Journal of Reproduction and Development, Vol. 46, No. 3, 2000

\title{
Histochemistry and Ultrastructure of the Intraluminal Mucus in the Sperm Reservoir of the Pig Oviduct
}

\author{
M. JOHANSSON'1), P. TIENTHAI') and H. RODRIGUEZ-MARTINEZ1,2)
}

\author{
Departments of ${ }^{1)}$ Anatomy and Histology and of ${ }^{2)}$ Obstetrics and Gynaecology, \\ Faculty of Veterinary Medicine, Swedish University of Agricultural Sciences (SLU), \\ Box 7039, SE-750 07 Uppsala, Sweden
}

\begin{abstract}
The presence of viscous mucus in some mammalian oviducts has been related to the arrest of spermatozoa on their ascent to the site of fertilization. This study describes the distribution of intraluminal mucus in the porcine oviductal sperm reservoir during estrus, using histochemistry and electron microscopy, including high resolution cryo-scanning electron microscopy (Cryo-SEM) of frozen tissue samples. The luminal contents and apical epithelium stained with Alcian blue (AB) and periodic-acid Schiff (PAS), were most conspicuous in the lower isthmus of pre-ovulatory females. The SEM-observations of glutaraldehyde/AB-fixed specimens confirmed the presence of a conspicuous intraluminal material, most prominent in the sperm reservoir of pre- and periovulatory animals. Such intraluminal material was consistently undetectable in glutaraldehydefixed, conventionally processed materials. In Cryo-SEM, a homogenous mass obliterated the lumen, especially in the pre-ovulatory UTJ/lower isthmus, diminishing towards the ampulla. Postovulation, the isthmic lumen appeared wider, showing only patches of amorphous material. If water sublimation was allowed during Cryo-SEM, the homogeneity of the luminal contents disappeared, leaving fibrillar remnants associated with the cilia and microvilli. The results clearly demonstrate the presence of luminal mucus in restricted areas of the pig oviduct, the pre-ovulatory sperm reservoir in particular, diminishing after ovulation. Its implication in the arrest and release of viable spermatozoa for fertilisation is discussed.
\end{abstract}

Key words: Intraluminal mucus, Cryo-electron scanning microscopy, Sperm reservoir, Oviduct, Pig.

(J. Reprod. Dev. 46: 183-192, 2000)

$\mathbf{G}$ amete transport, fertilization and early embryonic development are functions of the uterine tubes (oviducts). Although gamete and embryo transport have been associated with the myosalpingeal and ciliary activities of the organ [1], the intraluminal milieu plays a major role in all the essential reproductive events listed above [2]. The oviductal lumen contains a specific intraluminal fluid, built up by transudation from the vascular-rich lamina propria and by specific

Accepted for publication: April 6, 2000

Correspondence: H. Rodriguez-Martinez secretion by the lining epithelium [2, 3]. The gametes and the zygotes are affected by this intraluminal fluid during their transport through the oviduct [3].

The oviduct of the pig is clearly anatomically and physiologically regionalized, with different segments of the oviduct having different functions. The infundibulum and ampulla transport the newly ovulated oocytes and the ampullary-isthmic junction (AIJ) provides the site for fertilization to occur. The uterotubal junction (UTJ) and the adjacent first segment of the isthmus, on the other hand, act as modulators of sperm transport [4]. 
When spermatozoa reach this restricted area of the oviduct, they are arrested - establishing a sperm reservoir - for up to 36-40 h [5]. Here, most spermatozoa maintain normal ultrastructure and viability, escaping the attack of the immune system of the female [6,7]. Capacitation is believed to occur in vivo in the reservoir [8]. At the time around ovulation, some spermatozoa are released from this $\mathrm{UTJ} /$ isthmic reservoir, thus regulating the number of competent spermatozoa that might reach the oocytes at the AIJ [7, 9-11].

Several mechanisms have been proposed to explain the formation of the pre-ovulatory sperm reservoir (reviewed by [12]), including the mechanical obstruction of the lumen by intraluminal mucus $[13,14]$. Before ovulation, the isthmus segment of the oviduct of several mammals - such as in the rabbit [13], human [15] and the bovine species [14] - contains large amounts of a viscous mucoid secretion. This secretion, rich in glycoproteins [16], has been implicated in the arrest of spermatozoa [17] and ova transport [13, 18].

The aim of the present study was to examine the lumen of the porcine oviduct during estrus, in specimens retrieved at well determined pre- and post-ovulatory stages and prepared so that morphological preservation could be assured as well as to precipitate and fix extracellular mucus secretions in situ. It particularly aimed to describe the distribution of intraluminal mucus in the sperm reservoir during estrus, using histochemistry and electron microscopy, including high resolution cryo-scanning electron microscopy (cryo-SEM) of frozen tissue samples.

Preliminary observations have been reported elsewhere [19].

\section{Materials and Methods}

\section{Animals}

The experimental animals were sexually mature, cycling gilts $(\mathrm{n}=10)$ or pluriparous ( $2-4$ parities) sows $(n=10)$ (Swedish Yorkshire $x$ Swedish Landrace). The gilts had all shown at least one normal estrous cycle and their body weights ranged from 90 to $110 \mathrm{~kg}$, while the sows were aged between 1.5 and 2.5 years. All animals were, upon arrival (the sows immediately after weaning), allocated to individual pens in the vicinity of a fertile boar. The animals were given water ad libitum and commercial ration and were checked for standing estrus in the presence of a boar, by experienced personnel, twice daily. Half of the animals were either mated or artificially inseminated with $100 \mathrm{ml}$ of undiluted, raw semen (solely the sperm-rich fraction) from either of two fertile boars, on the first day of estrus (approximately 8-16 $\mathrm{h}$ after the first detection of standing estrus). The gilts were thereafter slaughtered either on the same day (day 1) or the following day (day 2) of standing estrus. The sows were slaughtered at well-defined pre- or postovulation stages $\pm 6-8$ hours from ovulation), as determined by transrectal ultrasonography [20]. The internal genitalia were promptly removed and the ovarian status was confirmed by visual examination. The experimental design was approved by the Ethical Committee for Experimentation with Animals, Sweden.

\section{Sampling and fixation}

The oviducts were immediately fixed either by:

1-Vascular perfusion, through the mid-uterine artery (4 gilts and 4 sows). One oviduct was selected at random, and perfused with a solution of 3\% glutaraldehyde in $0.067 \mathrm{M}$ sodium cacodylate buffer ( $\mathrm{pH}=7.2-7.3,500 \mathrm{mOsm})$. The contralateral oviduct was firstly perfused with a solution of $1.5 \%$ glutaraldehyde in $0.1 \mathrm{M}$ sodium cacodylate buffer (pH 7.4, $450 \mathrm{mOsm}$ ) for $5 \mathrm{~min}$ and then with a solution containing $1 \%$ of Alcian blue (AB, freshly prepared and filtrated) in the same buffer, according to the method described by [21].

2- Freezing, with or without cryoprotectant (6 gilts and 6 sows). The oviducts (including the entire UTJ) were dissected free from the mesosalpinx. One oviduct from each animal was fixed at random by plunging into liquid nitrogen $\left(\mathrm{LN}_{2}\right)$; while the contralateral oviduct was divided into segments (UTJ, lower-, mid- and upper-portions of the isthmus, AIJ, mid- and upper portions of the ampulla). From these segments, $5 \mathrm{~mm}$-long subsegments were frozen by direct plunging into $\mathrm{LN}_{2}$ with or without mounting on cryostat chucks using O.C.T. Compound (Tissue-Tek, Sakura Finetek USA Inc., Torrance, USA). A sample from the endocervix was always removed and processed as described above to serve as a positive control tissue for mucus histochemistry. The samples were kept in cryo vials (Nunc, Denmark) and stored in $\mathrm{LN}_{2}$ until further processing. 


\section{Histochemical studies}

The deep frozen tubal samples were cut on a cryostat (Leica CM 1800, Germany) into 5 and 10 $\mu \mathrm{m}$-thick transverse sections and mounted on polyL-lysine-coated slides (Polysine Microslides, Menzel-Gläser, Germany). The sections were left at room temperature $(20 \mathrm{C})$ for $60 \mathrm{~min}$ and then stained with periodic-acid-schiff (PAS) and/or Alcian blue (AB, Sigma, USA) at $\mathrm{pH} 2.5$, according to the method described by [16]. All sections were, after staining, dehydrated through a graded ethanol series, cleared in xylene and mounted with DPX (Kebo Lab, Sweden) before being examined on a Nikon Microphot-FXA light microscope (Nikon, Chiba, Japan).

\section{Ultrastructural studies}

The oviduct specimens were either processed for transmission electron microscopy (TEM) or scanning electron microscopy (SEM). The latter instrument was used for conventional SEM (chemically fixed samples) or for high resolution Cryo-SEM (frozen samples). For TEM, selected tubal segments (see above) including the endosalpinx, were trimmed into $1 \mathrm{~mm}^{3}$ pieces, rinsed in sodium cacodylate buffer $(0.067-0.1 \mathrm{M}$ at $\mathrm{pH} 7.2-7.4)$, post-fixed in $2 \%$ osmium tetroxide in sodium cacodylate buffer for $2 \mathrm{~h}$, rinsed in the buffer, dehydrated and embedded in Agar ${ }^{100}$ plastic resin (Agar-Aids, Essex, UK). Semithin sections (1 $\mu \mathrm{m})$ were cut and stained with saturated, boraxbuffered toluidine blue dye solution and were mounted and examined under light microscopy for selection of areas suitable for further fine sectioning. Once selected, ultra-thin sections were cut from selected areas with a diamond knife on a LKB Ultramicrotome (Bromma, Sweden), picked up on copper grids and stained with uranyl acetate and lead citrate. The sections were examined in a Philips EM 420 transmission electron microscope at $60 \mathrm{kV}$. For conventional SEM, selected tubal segments (see above) were trimmed to expose the lumen. Samples were then post-fixed in 1\% osmium tetroxide for $2 \mathrm{~h}$, dehydratred in increasing concentrations of acetone, critical point dried using carbon dioxide, mounted on stubs and sputtered with gold and palladium. Preparations were then examined in a Cambridge 150 (Cambridge, UK) scanning electron microscope at $20 \mathrm{kV}$. For CryoSEM, tubal segments were fractured within nitrogen vapour (approx. - $150 \mathrm{C}$ ) and held in a clamp-type specimen holder so that the endosalpinx (particularly the interfold areas) was exposed. The frozen sample was placed in the cryo stage (Oxford CT1500 HF, Oxford Instruments, Oxon, UK) and fractured with a scalpel blade to get a new, uncontaminated surface. A slight sublimation of the surface water was performed in order to visualize surface structures, by increasing the temperature from $-140-130 \mathrm{C}$ to $-90 \mathrm{C}$. After the sublimation, the sample was cooled down again to $-140 \mathrm{C}$ and sputtered with a $3 \mathrm{~nm}$ thin layer of chromium in order to avoid charging effects during examination in the microscope. After sputtering, the sample was transferred into a JEOL 6320F scanning electron microscope (JEOL Ltd, Tokyo, Japan) and examined at $5 \mathrm{kV}$.

\section{Results}

\section{Histochemical studies}

The cross sections of the frozen specimens showed a lumen that appeared very narrow compared to the embedded samples, particularly among those of the isthmus collected from preovulatory females (compare Figs. 1-2 and 3-4). The frozen sections of post-ovulation isthmic samples, however, presented a lumen that appeared much wider than pre-ovulation, including that of the isthmus (Fig. 3). Moreover, the pre-ovulatory lumen was filled with a material that stained with PAS and/or Alcian Blue (AB), was particularly conspicuous in the sperm reservoir (UTJ-isthmus, Figs 1-4). Spermatozoa were also evident in the sections of the sperm reservoir. Spermatozoa appeared immersed in conglomerates in the narrow deep furrows that characterized this tubal segment (Fig. 3), during pre-ovulation, whereas they were scattered as isolated spermatozoa in some postovulatory samples (Fig. 4). No obvious differences in qualitative histochemistry or sperm location were seen between the gilts and sows.

\section{Light microscopy of the sperm reservoir}

The porcine sperm reservoir consisted primarily of the crypts and furrows of the UTJ that continued with the deep areas of the longitudinal primary plicae (primary folds) of the endosalpinx in the adjacent isthmic segment, as seen in plastic semithin sections of control (glutaraldehyde-fixed) 

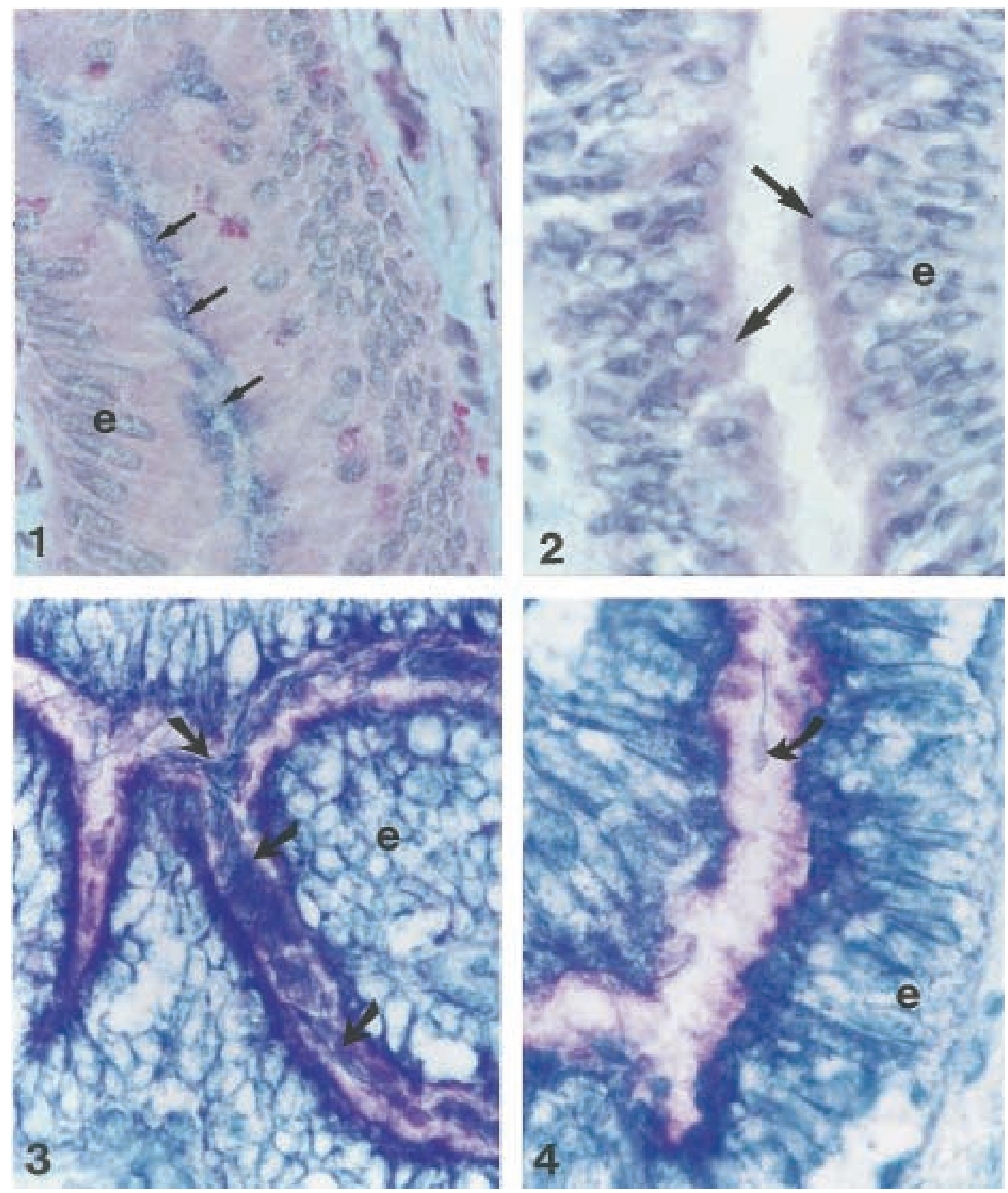

Figs. 1-4. Photomicrographs of frozen sections of the lower isthmus of pre-ovulatory $(1,3)$ and post-ovulatory $(2,4)$ pigs, either non-inseminated (1-2) or post-insemination (6 h, 3; 24 h, 4), stained with PAS and/or Alcian Blue (AB). In 1 and 3 , the lumen is very narrow and is filled with mucus (arrows, e: epithelium) stainable by both PAS and $A B$, and where spermatozoa are seen immersed in conglomerates (arrows in 3 ) in the deep furrows of the sperm reservoir. Note the larger lumen present in the post-ovulatory specimens $(2,4),(\times 500)$. 
samples of inseminated animals. The lining epithelium was continuous along the segments and consisted of two distinct cell types: ciliated and non-ciliated. The lining epithelium was separated from the glandless underlying lamina propria by a simple and thin basal lamina. Both cell types were easily distinguished by the unequal density of their cytoplasm, the presence of apical secretion granula or the presence of ciliae on the apical surface. Apart from these cell types, round lymphocyte or monocyte-like cells, were occasionally seen intermingled with the epithelial cells, particularly on the basal areas of the lining epithelium. The ratio of ciliated vs secretory cells (counted on semithin sections upon 500 epithelial cells/section, using an ocular reticulum) seemed to indicate that a larger number of secretory cells $(67 \%)$ was present in the reservoir than in the upper areas of the oviduct ( $51 \%$ of secretory cells in the ampulla). No obvious differences in the morphology (at light microscopy levels) of the sperm reservoir were determined between the inseminated and noninseminated females, nor between the gilts and sows or, among sows, in relation to parity. The majority of spermatozoa were, as expected, seen as clusters in the crypts of the UTJ and in the deep furrows of the isthmus segment. They were also found, individually or in small conglomerates, in the central lumen of the organ and the inter-fold areas. In these control specimens, the lumen was evident and free from debris, except for the spermatozoa present, sometimes attached to the apical ciliae or microvilli. No obvious qualitative differences in sperm location (no quantitation attempts were made) were seen between specimens collected at pre- or post-ovulation.

\section{Ultrastructural studies}

Scanning Electron Microscopy (SEM): The control samples prepared for SEM showed clearly that the lining epithelium consisted of non-ciliated cells (covered with microvilli) intermingled with nonciliated cells, either as individual cells or small cell groups (Fig. 5). There was a striking difference between the samples fixed by vascular perfusion with glutaraldehyde and those fixed with glutaraldehyde $/ A B$, particularly in the preovulatory specimens. The glutaraldehyde/ABfixed samples presented a conspicuous amorphous material in the lumen, masking the apical surface of the lining epithelium (compare Figs. 5 and 6).
This amorphous material (as seen in SEM) corresponded, in location, to the intraluminal mucus previously detected in the histochemistryprepared samples and examined with light microscopy (see Histochemical studies). The postovulatory samples, however, presented lower amounts of this intraluminal mucus cloud (mostly present as fragmented clouds in some specimens). No obvious differences in location and amount of intraluminal material were noticed between noninseminated and inseminated specimens, and neither between the gilts and sows.

Transmission Electron Microscopy (TEM): Complementary electron micrographs of the pig isthmus, conventionally prepared for TEM, confirmed the absence (Fig. 7$)$ or presence $\left({ }^{*}\right.$ in Fig. 8) of this intraluminal mucus among and above the cilia and microvilli. Here again, no major differences were noticed between non-inseminated and inseminated specimens, nor between gilts and sows. The intraluminal mucus was less noticeable in the post-ovulatory samples.

Cryo-SEM: When quickly frozen and prepared for Cryo-SEM, the pre-ovulatory samples showed a rather homogenous intraluminal material that obliterated their lumen (Figs. 9 and 11). Here, spermatozoa were seen immersed in this intraluminal material (arrows in Figs. 11 and 12). The lumen appeared wider post-ovulation. A fibrillar material was more clearly seen when slight sublimation was practiced inside the cryo-chamber of the microscope (Fig. 10). No differences were hereby seen, regarding the appearance of the intraluminal material, between the non-inseminated or inseminated specimens, nor between gilts and sows.

\section{Discussion}

The present study determined the presence of intraluminal mucus in the sperm reservoir of the pig during estrus, as shown using histochemical and electron microscopical methods. The intraluminal mucus was conspicuous during preovulation. It obliterated the very narrow lumen at pre-ovulation, but it was less obvious when ovulation had spontaneously occurred, and the lumen became wider in most specimens. Spermatozoa were immersed in this intraluminal material, as clearly seen in the pre-ovulatory 

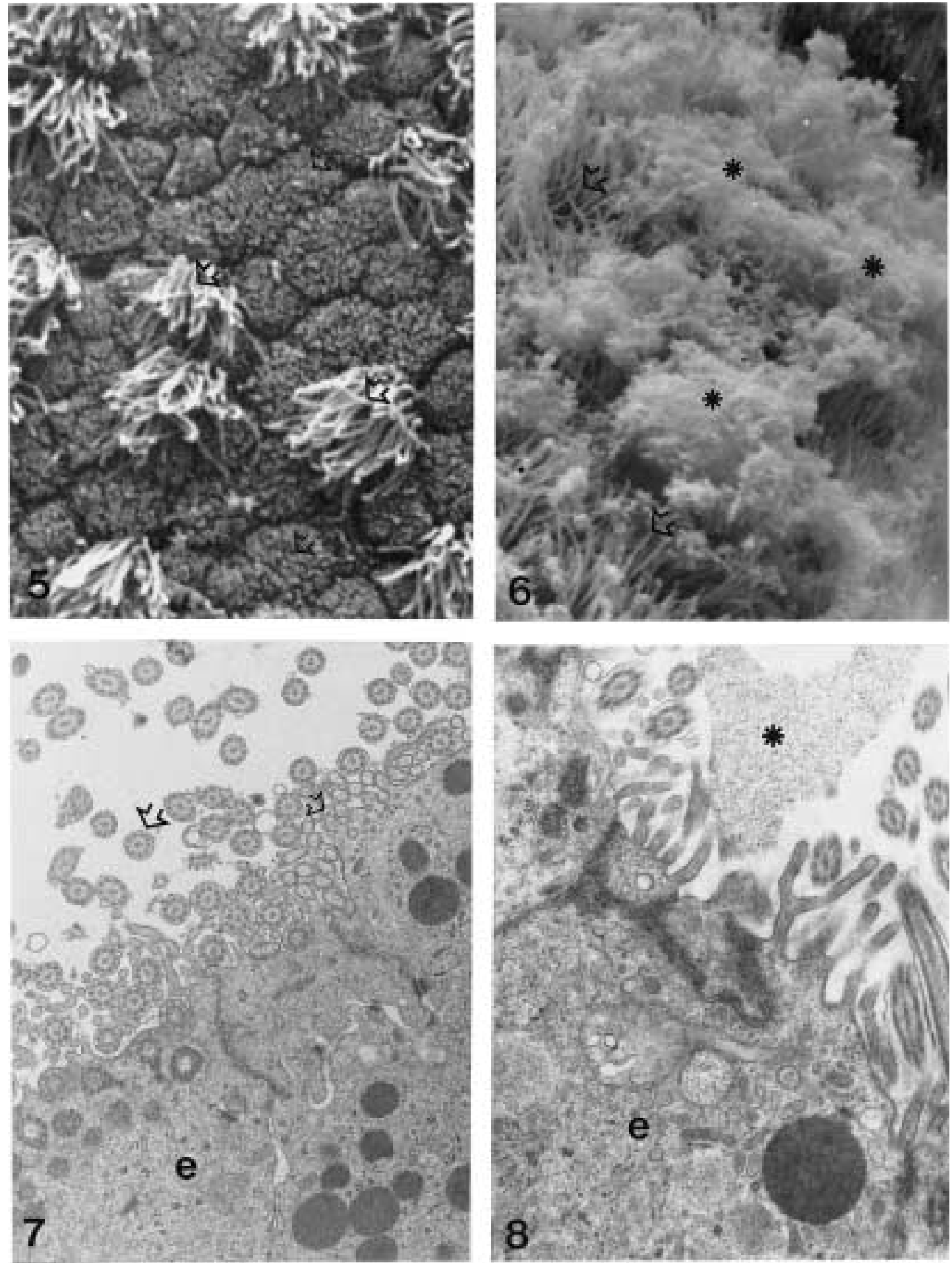

Figs. 5-8. Electron micrographs (conventional SEM $(5-6 ; \times 2,000)$ and TEM $(7-8 ; \times 20,000)$ of chemicallyfixed samples) of the pig isthmus showing the presence of intraluminal mucus $\left(^{*}\right)$ in the intraluminal milieu (e: epithelium). In Fig. 5, a control glutaraldehyde-fixed pre-ovulatory isthmus where barely any mucus is visible (large open arrows: ciliated cells, small open arrows: secretory cells). In Fig. 6, the contralateral isthmus - following fixation with glutaraldehyde-Alcian blue - shows an amorphous material in the lumen. Figures 7 and 8 are TEM sections of the same organs. 

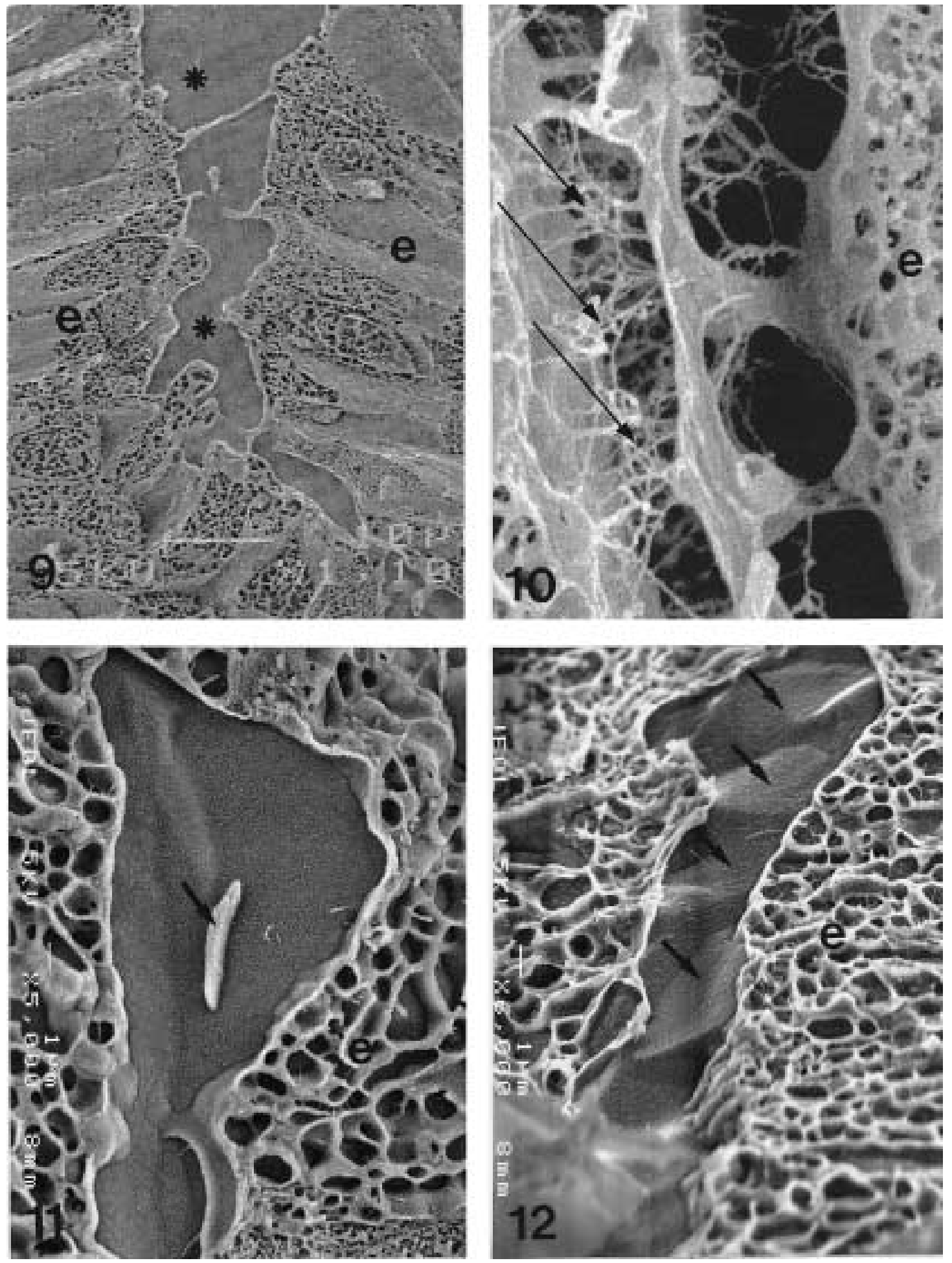

Figs. 9-12. Cryo-SEM micrographs (deep-frozen samples) of the porcine oviductal isthmus collected preovulation (Figs. 9, 11-12) $6 \mathrm{~h}$ post insemination and a post-ovulatory sample (10). Compare the intraluminal contents in Fig $9(\times 1,000)$ and $10(\times 11,000)$. The compact mucus obliterates the lumen during pre-ovulation $\left(^{*}\right)$ while the lumen appears wider post-ovulation with a fibrillar material (long arrows). Spermatozoa (arrows) are present inside the intraluminal contents either singly $(11 ; \times 5,000)$ or in groups $(12 ; \times 6,000)$, e: epithelium. 
samples.

The presence of mucus-like material in the lumen of the lower oviduct has been reported in other species such as the rabbit [13], human [15] and bovine [14], irrespective of the presence of spermatozoa. In the pig, the amount of mucus was more conspicuous during the pre-ovulatory period in relation to the changes in secretory activity of the lining epithelium [22-24]. The secretory activity of the epithelium depends upon the stage of the estrous cycle. There is general agreement that the secretory materials accumulate in the secretory cells during the follicular phase and that maximal secretion occurs around ovulation, the time when gametes and embryos are present in the oviduct [22]. The secretory activity in the porcine epithelium was stimulated by estrogen and decreased under the influence of progesterone $[25,26]$.

The oviductal epithelium is composed of ciliated and non-ciliated secretory cells. The non-ciliated cells synthesize and secrete specific glycoproteins (reviewed by [27]). The oviductal proteins are stored in vesicles or granules in the secretory cells until they are released to the oviductal lumen. These specific oviductal proteins are supposed to play an important role in fertilization and the early embryonic stages of development (reviewed by [3]), affecting the survival of the oocytes and embryos [28].

The nature of the mucus components observed in the present study is yet unknown. The mucus, probably represented by a variety of mucopolysaccharide species (including oviductal specific glycoproteins [29]), might participate in the in vivo pre-ovulatory arrest of spermatozoa, i.e. the formation of the sperm reservoir [30]. Alcian blue predominantly stains sulfated glycosaminoglycans (GAGs) [31] but not hyaluronan (HA). These GAGs have been identified in the isthmus of the porcine oviduct, using immunohistochemistry [12, 32, 33]. Variations in GAGs concentrations were also recently determined in oviductal fluid collected in vivo from sows [33]. The contents of GAGs in the fluid showed temporal variations, being higher during late proestrus and pre-ovulatory estrus [33].

As stated above, both PAS- and AB-stained mucoid material was present in areas of the pig oviduct in which spermatozoa were retained, awaiting ovulation. The present results show that, in contrast to the bovine, most spermatozoa in the pig UTJ-isthmic reservoir were located in the narrow deep furrows that characterize the porcine sperm reservoir. These deep-allocated spermatozoa are intact, and in contact with the endosalpinx as shown in tubal tissues fixed chemically and studied by conventional electron microscopy [6]. Using cryo-scanning electron microscopy (Cryo-SEM) of deep-frozen tubal samples, spermatozoa could be seen immersed in an amorphous intraluminal material in these deep furrows of the isthmic reservoir, not necessarily in direct contact with the epithelium. Cryo-SEM is a method that enables the in situ visualisation of the water-soluble components of the intraluminal fluid and therefore shows a less modified view of the organs, in biochemical terms. In vivo, the role of the sperm reservoir seems to be to allow a population of potentially fertile spermatozoa to be present and viable in the oviduct for a rather long period prior to ovulation that, in the pig, occurs rather late during estrus. The sperm reservoir also offers a filtering strategy so that only a small number of spermatozoa is allowed to travel to the site of fertilization up in the ampullary-isthmic junction, thereby reducing the chances for the occurrence of lethal polyspermy [10]. To consider that the mucus represents a sticky material that delays the displacement of spermatozoa along the oviductal reservoir is a tempting hypothesis, but it has yet to be tested. Obliteration of the lumen could also be responsible for the arrest of spermatozoa. Such obliteration could also be due to the edema of the lamina propria [4], the contractility of the myosalpix [1], or most probably, a combination of factors [12], all conveying a delay in sperm displacement.

The oviduct also appears to regulate the rate of the capacitation process in vivo $[8,34,35]$. Most evidence so far presented suggests that it is the attachment of spermatozoa to the apical epithelium, usually seen in conventional preparations $[6,7,9]$ or in explants [36], that modulates the rate of capacitation [36]. Attachment to the epithelium seems to keep intracellular levels of $\mathrm{Ca}^{++}$low, thus delaying the cascade of changes that characterizes capacitation, at least in the equine $[37,38]$. This process is still not yet fully understood, but stabilization of the sperm plasmalemma by the surrounding fluid might be involved in preventing passive $\mathrm{Ca}^{++}$inflow. This delay of capacitation might, in turn, extend the viability and fertilizing 
capacity of the spermatozoa [39]. In the light of the present observations, where most spermatozoa are seen immersed in the intraluminal fluid in situ when observed with Cryo-SEM, it remains to be determined how the intraluminal secretion, or the attachment of spermatozoa to the epithelium delay capacitation. An interesting hypothesis, circumstantially supported by our own results and presented elsewhere [12], is that boar spermatozoa are trapped in the tubal reservoir by the luminal mucus present in the narrow lumen. Capacitation, perhaps already programmed in the spermatozoa, is delayed or prevented by this mucus or by the attachment to the epithelium until modifications in hormonal status (not yet disclosed fully) or other factors change this milieu or trigger a relocation of sperm sub-populations from the reservoir. These sperm aliquots of the sperm population in the reservoir, mostly represented by capacitated, intact and potentially fertile spermatozoa, are then transported towards the site of fertilization, perhaps in connection with the hereby seen opening of the lumen, the disappearance of the mucus and the increase of specific myosalpingeal motility [1].

\section{Acknowledgements}

The authors acknowledge Marianne Ekwall and Hans Ekwall for excellent technical help. This study was supported by the Royal Swedish Academy for Forestry and Agriculture (KSLA), the Swedish Foundation for International Cooperation in Research and Higher Education (STINT), and the Swedish Council for Forestry and Agricultural Research (SJFR), Stockholm.

\section{References}

1. Rodriguez-Martinez $\mathbf{H}$, Einarsson S, Larsson $\mathbf{B}$. Spontaneous motility of the oviduct in the anaesthetized pig. J Reprod Fert 1982; 66: 615-624.

2. Leese HJ. The formation and function of oviduct fluid. J Reprod Fert 1988; 82: 843-856.

3. Gandolfi F. Functions of proteins secreted by oviduct epithelial cells. Microsc Res Techn 1995; 32: $1-12$.

4. Hunter RHF. Preovulatory arrest and periovulatory redistribution of competent spermatozoa in the isthmus of the pig oviduct. $J$ Reprod Fert 1984; 72: 203-211.

5. Viring S, Einarsson S, Nicander L, Larsson K. Localization of the sperm "reservoir" at the uterotubal junction of the pig. Proc 9th Int Congr Anim Reprod \& AI (Madrid) 1980; 3: 331.

6. Rodriguez-Martinez $\mathbf{H}$, Nicander $L$, Viring $S$, Einarsson S, Larsson K. Ultrastructure of the uterotubal junction in preovulatory pigs. Anat Histol Embryol 1990; 19: 16-36.

7. Mburu J, Rodriguez-Martinez $\mathbf{H}$, Einarsson S. Changes in sperm ultrastructure and localisation in the porcine oviduct around ovulation. Anim Reprod Sci 1997; 41: 137-148.

8. Smith TT, Yanagimachi R. Capacitation status of hamster spermatozoa in the oviduct at various times after mating. J Reprod Fert 1989; 86: 255-261.

9. Fléchon JE, Hunter RHF. Distribution of spermatozoa in the utero-tubal junction and isthmus of pigs, and their relationship with the luminal epithelium after mating: a scanning electron microscope study. Tissue Cell 1981; 13:
127-139.

10. Hunter RHF, Flechon B, Flechon JE. Pre- and periovulatory distribution of viable spermatozoa in the pig oviduct: a scanning electron microscopy study. Tissue Cell 1987; 19: 423-436.

11. Hunter RHF. Oviduct function in pigs, with particular reference to the pathological condition of polyspermy. Mol Reprod Dev 1991; 29: 385-391.

12. Rodriguez-Martinez $\mathbf{H}$, Larsson $B$, Pertoft $\mathbf{H}$, Kjellén L. GAGs and spermatozoon competence in vivo and in vitro. ICAR 50th Special Anniversary Conference "Gametes: Development and Function", Serono, Milano; 1998: 239-272.

13. Jansen RPS. Fallopian tube isthmus mucus and ovum transport. Science 1978; 201: 349.

14. Suarez SS, Brockman K, Lefevre R. Distribution of mucus and sperm in bovine oviducts after artificial insemination: the physical environment of the oviductal sperm reservoir. Biol Reprod 1997; 56: 447-453.

15. Jansen RPS. Cyclic changes in the human Fallopian tube isthmus and their functional importance. Am J Obstet Gynecol 1980; 136: 292308.

16. Menghi G, Bondi AM, Accili D, Materazzi G. Fine localization of sulfated and non-sulfated glycoconjugates in the rabbit oviduct during the estrous cycle. Acta Histochem 1984; 74: 121-132.

17. DeMott RP, Suarez SS. Hyperactivated sperm progress in the mouse oviduct. Biol Reprod 1992; 46: 779-785.

18. Jansen RPS, Bajpai VK. Oviduct acid mucus 
glycoproteins in the estrous rabbit: Ultrastructure and histochemisry. Biol Reprod 1982; 26: 155-163.

19. Johansson $\mathbf{M}, \mathbf{M b u r u} \mathbf{J}$, Rodrìguez-Martìnez $\mathbf{H}$. Ultrastructure of the endosalpinx and demonstration of oviduct luminal mucus in the pig. Proc 5th Int Conf Pig Reprod, Kerkrade, Holland, 1997; 88.

20. Mburu JN, Einarsson S, Dalin AM, RodriguezMartinez H. Ovulation, as determined by transrectal ultrasonography in multiparous sows: Relationships with oestrous symptoms and hormonal profiles. J Vet Med A 1995; 42: 285-292.

21. Behnke O, Zelander T. Preservation of intercellular substances by the cationic dye alcian blue in preparative procedures for electron microscopy. J Ultrastruct Res 1979; 31: 424-438.

22. Iritani A, Sato E, Nishikawa Y. Secretion rates and chemical composition of oviduct and uterine fluids in sows. J Anim Sci 1974; 39: 582-588.

23. Buhi WC, Alvarez IM, Sudhipong V, DonesSmith MM. Identification and characterization of the novo-synthesized porcine oviductal secretory proteins. Biol Reprod 1990; 43: 929-938.

24. Buhi WC, Alvarez IM, Choi I, Cleaver BD, Simmen FA. Molecular cloning and characterization of an estrogen-dependent porcine oviductal secretory glycoprotein. Biol Reprod 1996; 55: 1305-1314.

25. Nayak RK, Zimmerman DR. Ultrastructural changes in porcine oviduct epithelium during the estrous cycle. J Anim Sci 1971; 33: 262.

26. Nayak RK, Zimmerman DR, Albert EN. Electron microscopic studies of estrogen-induced ciliogenesis and secretion in uterine tube of the gilt. Am J Vet Res 1976; 37: 189-197.

27. Jansen RPS. Ultrastructure and histochemistry of acid mucus glycoproteins in the estrous mammal oviduct. Micr Res Techn 1995; 32: 29-49.

28. Buhi WC, O'Brien B, Alvarez IM, Erdos G, Dubois D. Immunogold localization of porcine oviductal secretory proteins within the zona pellucida, perivitelline space, and plasma membrane of oviductal and uterine oocytes and early embryos. Biol Reprod 1993; 48: 1274-1283.

29. Malette B, Paquette Y, Merlin Y, Bleau G. Oviductins possess chitinase- and mucin-like domains: a lead in the search for the biological function of these oviduct-specific ZP-associated glycoproteins. Mol Reprod Dev 1995; 41: 384-397.

30. DeMott RP, Lefebvre R, Suarez SS. Carbohydrates mediate the adherence of hamster sperm to oviductal epithelium. Biol Reprod 1995; 52: 1395-1403.

31. Lin W, Shuster S, Mailbach HI, Stern R. Patterns of hyaluronan staining are modified by fixation techniques. J Histochem Cytochem 1997; 45: 11571163.

32. Rodriguez-Martinez $\mathbf{H}$, Pertoft $\mathbf{H}$, Johansson $\mathbf{M}$. Cryo-scanning electron microscopy of the porcine oviduct and immunocytochemical localization of hyaluronan in the endosalpinx. Theriogenology 1998; 49: 335.

33. Tienthai P, Kjellén L, Pertoft H, Suzuki K, Rodriguez-Martinez $\mathbf{H}$. Localisation of hyaluronan and sulphated glycosaminoglycans in the pig oviduct: Immunohistochemistry and quantitation in intraluminal fluid. Submitted for publication.

34. Smith TT, Nothnick WB. Role of direct contact between spermatozoa and oviductal epithelial cells in maintaining rabbit sperm viability. Biol Reprod 1997; 56: 83-89.

35. Shalgi R, Smith TT, Yanagimachi R. A quantitative comparison of the passage of capacitated and uncapacitated hamster spermatozoa through the uterotubal junction. Biol Reprod 1992; 46: 419-424.

36. Fazeli A, Duncan AE, Watson PF, Holt WV. Sperm-oviduct interaction: induction of capacitation and preferential binding of uncapacitated spermatozoa to oviductal epithelial cells in porcine species. Biol Reprod 1999; 60: 879886.

37. Dobrinski I, Suarez SS, Ball BA. Intracellular calcium concentration in equine spermatozoa attached to oviductal epithelial cells in vitro. Biol Reprod 1996; 54: 783-788.

38. Dobrinski I, Smith TT, Suarez SS, Ball BA. Membrane contact with oviductal epithelium modulates the intracellular calcium concentration of equine spermatozoa in vitro. Biol Reprod 1997; 56: 861-869.

39. Murray SC, Smith TT. Sperm interaction with Fallopian tube apical membrane enhances sperm motility and delays capacitation. Fertil Steril 1997; 68: 351-357. 